\title{
Adaptive Stereo Matching in Correlation Scale-Space ${ }^{\star}$
}

\author{
Christian Menard and Walter G. Kropatsch \\ Pattern Recognition and Image Processing Group, \\ Vienna University of Technology, Treitlstraße 3/183/2, \\ A-1040 Vienna, Austria, e-mail: men@prip.tuwien.ac.at
}

\begin{abstract}
Stereo computes the distance of objects, "their depth", from two images of two cameras using the triangulation principle. Points of imaged objects are mapped in different locations in the two stereo images. A central problem in stereo matching using correlation techniques lies in selecting the size of the search window. Small windows contain only a small number of data points, and thus are very sensitive to noise and therefore result in false matches. Whereas large search windows contain data from two or more different objects or surfaces, thus the estimated disparity is not accurate due to different projective distortions in the left and the right image. The new method introduces a continuous scale parameter for the matching process. It allows the adaption of the scale for every individual region and overcomes the drawbacks of fixed window sizes which is impressively demonstrated by the experimental results.
\end{abstract}

\section{Introduction}

In order to reconstruct the three-dimensional information of a scene, computer vision uses two different views of the same scene. This technique is known under the term stereo-vision or binocular vision. It is important for a vision system that interesting structures for certain scales are found by the stereo-vision process. According to Barnard and Fischler [1] a stereo-vision system performs the following steps: image acquisition, camera calibration, extraction of points of interest, depth determination, and depth interpolation.

All of these steps play important roles in the design of a stereo system and it is of interest to know how the different tasks depend on each other. Even small mistakes that are made during this processes can result in wrong depth information for the object currently under consideration. Starting from the image acquisition, noise can be introduced for certain reasons into the images, which complicates the modeling of the used cameras and, of course, the search for the corresponding points in a stereo pair. But the success of the approach mostly depends on its ability to solve the problem of stereo matching, a topic upon which this work concentrates.

The paper is organized as follows: In the next section we review basic notation of area-based stereo matching. Section 3 discusses the problem of the window size. A new adaptive matching method is proposed using a correlation scalespace. Experimental results are described in section 4 . Finally we give a brief summary.

\footnotetext{
* This work was supported by the Austrian Science Foundation (FWF) under the
} grant Nr. P09954-SPR and Nr. S7002-MAT. 


\section{Standard Area-Based Stereo Matching}

The epipolar geometry is the starting point for this method. For a given pair of stereo images, the corresponding points are supposed to lie on the epipolar lines [10]. Since a parallel camera alignment is used in this paper, the epipolar lines are the scanlines in both images. In Fig. 1 a synthetic stereo pair is depicted

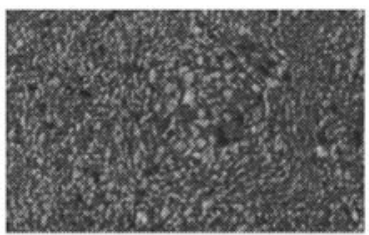

(a) left image

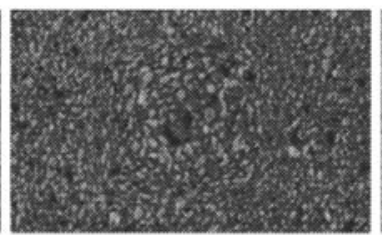

(b) right image

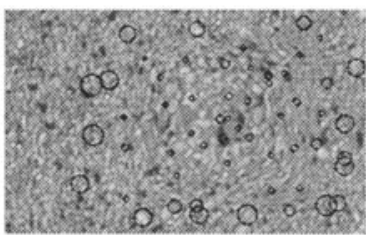

(c) optimal scales

Fig. 1. Synthetic stereo pair: Pyramid on a flat ground with natural texture added on the surface. (c) optimal scales for points of interest.

which consists of a pyramid on a plane ground with natural texture added on the surface. If for a given point $\left(x_{L}, y_{L}\right)$ in the left image, a corresponding point $\left(x_{R}, y_{R}\right)$ in the right image can be found, the three dimensional position of the object point can be computed with the additional information about the camera parameters. As a similarity measure the correlation of gray-level intensities is used. 1D-correlation $C\left(x_{L}, x_{R}, w\right)$ between two intervals of size $w$ can be written as convolution with a rectangular function $\delta_{1 / w}[8,6]$.

$$
\begin{gathered}
C\left(x_{L}, x_{R}, w\right)=\frac{\left[I_{L}\left(x_{L}\right) I_{R}\left(x_{R}\right)\right] * \delta_{1 / w}-\mu_{L}\left(x_{L}, w\right) \mu_{R}\left(x_{R}, w\right)}{\sqrt{\left[I_{L}^{2}\left(x_{L}\right) * \delta_{1 / w}-\mu_{L}^{2}\left(x_{L}, w\right)\right]\left[I_{R}^{2}\left(x_{R}\right) * \delta_{1 / w}-\mu_{R}^{2}\left(x_{R}, w\right)\right]}}, \\
\quad \text { with } \mu(x, w)=I(x) * \delta_{1 / w}(x), \text { where } \delta_{1 / w}(x)=\left\{\begin{array}{cc}
\frac{1}{w} & |x| \leq \frac{w}{2} \\
0 & \text { elsewhere }
\end{array} .\right.
\end{gathered}
$$

The 2D-correlation $C\left(x_{L}, y_{L}, x_{R}, y_{R}, w\right)$ for two-dimensional regions can easily be extended from equation (1). For each point in the left stereo image the disparity information $D\left(x_{L}, y_{L}\right)$ is computed using the correlation function $C$ as

$$
D\left(x_{L}, y_{L}\right)=\left\{\begin{array}{cl}
x_{L}-x_{R} & \text { for } x_{R}=\operatorname{argmax}\left\{C\left(x_{L}, y_{L}, x_{R}, y_{R}, w\right)\right\}>T \\
-1 & \text { otherwise }
\end{array},\right.
$$

where $T$ defines the minimal threshold for accepting a corresponding point. The fact that the maximum of $C$ is below this threshold $T$ may be caused by occlusion, highlights or depth discontinuities. The maximum of the correlation function is accepted if it is above the threshold $T$. In this case the position of the distinct maximum defines the corresponding point in $I_{R}$.

A central problem in finding correspondences lies in selecting the size of the search window $w$. Small windows contain only a small number of data points, and thus are very sensitive to noise and therefore result in false matches. Whereas 
large search windows contain data from two or more different objects or surfaces, thus the estimated disparity is not accurate due to different projective distortions in the left and the right image. The strategy is to find a corresponding point with the smallest window size $w$. There exist various works dealing with this problem; Levine et al. use an adaptive correlation window, the size of which varies inversely with the variance of the region which is currently considered [4]. In another adaptive approach Kanade and Okutomi proposed that the size and shape of the matching window is chosen adaptively on the basis of local evaluation of the variation in both the intensity and the disparity [3]. In all these works the search window is rectangularly shaped and the size is changed in a discrete way.

In the next section a new method is proposed by changing the size of the window in a continuous way, thus making it possible to determine an optimal size for a given region.

\section{Adaptive Stereo Matching}

The problem of changing the size of the search window during the matching process depends on the objects which are considered. If a window contains data from two or more objects or surfaces the correlation for that region does not show a clear maximum, unless the window is decreased and contains only data points from one single object. Another problem occurs when a search window contains occluded regions. In this case the computed disparity value is not correct. In order to find an optimal size of the search window the function $C$ has to be modified in such way that the scale can be changed in a continuous way.

\subsection{Gaussian Weighted Correlation}

The products of intensity values in equation (1) are constantly weighted with the normalizing function $\delta_{1 / w}(2)$. The formulation as convolution allows us to substitute $\delta_{1 / w}$ by the Gaussian weights. In the following, the scale-space notation ${ }^{2}$ is used, where the scale is defined by $t$. For a $1 \mathrm{D}$-image $I: \mathbb{R} \mapsto[0,1]$ we define the Gaussian weighted local mean $\mu: \mathbb{R} \times \mathbb{R} \mapsto[0,1]$

$\mu(x ; t)=\int_{-\infty}^{\infty} I(x-\xi) g(x+\xi, t) d \xi=I(x) * g(x ; t)$, with $g(x ; t)=\frac{1}{t \sqrt{2 \pi}} e^{\frac{-x^{2}}{2 t^{2}}}$.

The Gaussian weighted standard deviations are defined by

$$
\sigma_{k}^{2}(x ; t)=I_{k}^{2}(x) * g(\cdot ; t)-\mu_{k}^{2}(x ; t) \quad k=L, R,
$$

and the covariance can be written as

$$
\sigma_{L R}^{2}\left(x_{L}, x_{R} ; t\right)=\left[I_{L}\left(x_{L}\right) I_{R}\left(x_{R}\right)\right] * g(\bullet ; t)-\mu_{L}\left(x_{L} ; t\right) \mu_{R}\left(x_{R} ; t\right) \quad k=L, R .
$$

The correlation can be written as convolution with the Gaussian kernel in the one-dimensional case (4) as follows:

$$
C_{\Gamma}(\cdot ; t)=\frac{\left[I_{L}\left(x_{L}\right) I_{R}\left(x_{R}\right)\right] * g(\cdot ; t)-\mu_{L}\left(x_{L} ; t\right) \mu_{R}\left(x_{R} ; t\right)}{\sqrt{\left[I_{L}^{2}\left(x_{L}\right) * g(\cdot ; t)-\mu_{L}^{2}\left(x_{L} ; t\right)\right]\left[I_{R}^{2}\left(x_{R}\right) * g(\cdot ; t)-\mu_{R}^{2}\left(x_{R} ; t\right)\right]}} .
$$

${ }^{2}$ The notation $C_{\Gamma}(\bullet ; t)$ stands for $C_{\Gamma}\left(x_{L}, x_{R} ; t\right)$ and $C_{\Gamma}(\bullet, \bullet ; t)$ for
$C_{\Gamma}\left(x_{L}, y_{L}, x_{R}, y_{R} ; t\right)$. 
For the two-dimensional case the two-dimensional Gaussian kernel is used and the 2D-weighted correlation function $C_{\Gamma}(\bullet, \bullet ; t)$ can be extended from equation (7). Instead of using all the data points in the correlation window equivalently weighted, the window is weighted with the Gaussian kernel. The size of the search window can be controlled by the scale parameter $t$. The influence of pixels far from the center of the window diminish at a rate controlled by $t$. Furthermore the shape of the search window has changed from rectangular to circular. The function $C_{\Gamma}$ defines a Correlation Scale-Space (CSS) for one point $I_{L}(x, y)$ in the left stereo image. In the $C S S$ the similarity value is available at different scales driven by the parameter $t$ of the scale-space kernel. The main advantages of the CSS compared to standard methods, such as the hierarchical approach, is that the scale can be changed in a continuous way. Furthermore in this representation all levels of scale are immediately accessible.

\subsection{Optimal Scale Selection}

In general situations it is not possible to know in advance at what scales interesting structures can be expected to appear. Size variations of image structures in a stereo pair can occur for several reasons:

- objects in the scene have different physical size;

- surface textures contain structures at different scales; and

- scale variations appear due to perspective distortions.

There are many ways to select the best scale for a given problem. A very interesting work in this field was presented by Lindeberg in which he describes the "scale-space primal sketch" [5]. An operator gives maximal output if its size is best tuned to the object. Other approaches study the variation of the information content over scale [2]. For the correspondence establishment it is possible so far to change the scale parameter $t$ in a continuous way using the correlation function $C_{\Gamma}$. But the problem to be solved is to find the "best scale(s)" $t_{\text {opt }}$ for certain regions in a stereo pair. Basically, the scale at which a maximum over scales is attained will be assumed to give information about the window size for that region. The maximum over scale for a region defines the optimal scale. In the next step the CSS for different placements of a point is analyzed: on a plane parallel to the image plane; on a roofed surface; near a depth edge; and in an occluded area. For these situations the correlation values at the corresponding

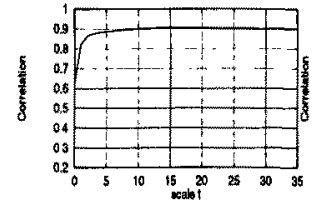

(a) plane

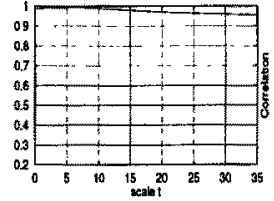

(b) roofed object

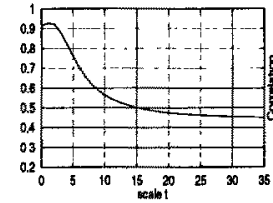

(c) depth edge

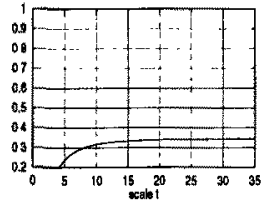

(d) occluded area

Fig. 2. Optimal scale values for different situations: (a) $C_{\Gamma}=0.91$ at $t=15.0$, (b) $C_{\Gamma}=0.99$ at $t=3.0$, (c) $C_{\Gamma}=0.92$ at $t=1.5$ and (d) $C_{\Gamma}=0.35$ at $t=35$.

position in the right stereo image are tested by tracing along the local maxima in the direction of the scanline from high to low scale. In Fig. 2 the correlation 
function at the corresponding point along scale $t$ is visualized for these different situations.

Plane: For the plane lying parallel to the image plane there are only small variations along the correlation function. The correlation value decreases if no or not enough gray-level information is available in the correlation window. The scale-space maximum for this example lies at $t=15$, with $C_{\Gamma}(\bullet, \bullet ; t) \in[0.6 .0 .9]$, which means that for this scale the correlation value can be maximized.

Roofed surface: In the case of a roofed surface, by tracing from large to smaller scale the value of the correlation increases successively until it decreases because of too little information in the correlation window. For this example a small scale obtains the highest correlation value at scale $t=3$, with $C_{\Gamma}(\bullet, \bullet ; t) \in[0.95 . .0 .99]$.

Depth Edge: Near a depth edge it is similar. At larger scales the correlation value is low because the window contains data from two objects which have different disparity values. By decreasing the scale parameter $t$ the correlation value is maximized at a very low scale, since the window contains only data from one single object. The obtained scale for this situation lies at $t=1.5$, with $C_{\Gamma}(\bullet, \bullet ; t) \in[0.45 . .0 .93]$.

Occluded area: In the last test the correlation value over scale for an occluded region is determined. It can be seen in Fig. 2 (d) that the maximum of the correlation function along scale is at $t=35$ with $C_{\Gamma}(\bullet, \bullet ; t) \in[-0.1 .0 .35]$. This represents the highest scale and the correlation value decreases successively to lower scale. But the correlation value is very low anyway, thus the position of this maximum represents an average depth information of the surrounding regions, which contain the occluded area.

In order to establish correspondences, the function $C_{\Gamma}$ is tested on the pyramid (Fig. 1). In the left image some points of interest are chosen for which the corresponding points are determined in the right stereo image. Every scale-space maximum is graphically illustrated by a circle centered at the point in the left stereo image for which the correspondence is established. The size of the circle corresponds to the scale of maximum correlation. The circles are superimposed on a bright copy of the left image. The result is visualized in Fig. 1 (c). The optimal scale of points on the surface of the pyramid is small, since the planes form an oblique angle with the image plane, thus not all points in the search window have the same disparity value, whereas for regions on the flat ground the selected scale value is large. One way to determine the optimal scale $t_{\text {opt }}$ for finding correspondences is to determine the scale-space maximum by tracing from high to low scale along the correlation maxima. By following the correlation maximum from high to low scale a change in the direction $x_{R}$ defines a variation in the disparity value. The algorithm can be outlined:

1. Compute the initial disparity value $D_{0}$ using the correlation function $C_{\Gamma}$ starting at a large scale $t_{0}=t_{\text {max }}$. If no unique maximum can be determined there is no corresponding point.

2. Decrease the scale with $t_{n+1}=t_{n} / \Delta t$

3. Compute the new disparity value $D_{n+1}$ by using the previously estimated disparity $D_{n}$.

4. Iterate steps 2, and 3. until a global maximum along the scale is found or a maximum number of iterations is reached. 


\section{Experimental Results on Synthetic and Real Images}

The adaptive matching algorithm using function $C_{\Gamma}$ is applied on two synthetic and real images and is compared to the standard stereo approach.

As synthetic stereo pairs a pyramid and a sphere on a flat ground are used in each case with texture added onto the surface. The disparity values for these synthetic sets of stereo pairs are known to compare the absolute accuracy of the matching method. For each test the accuracy is compared to the results

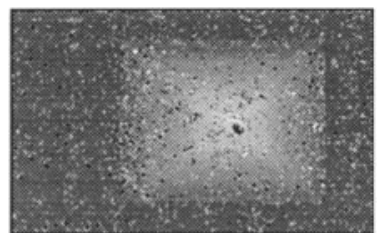

(a) with $C, w=3$

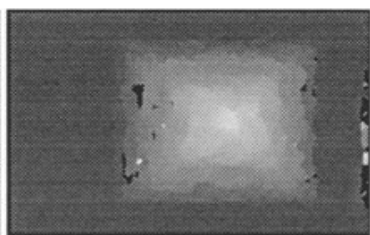

(b) with $C, w=15$

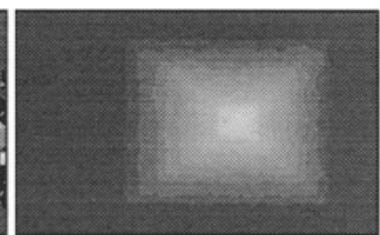

(c) with $C_{\Gamma}$

Fig. 3. Pyramid on a plane ground with a natural texture added onto the surface: (a) (b) disparity maps computed with the standard stereo method using fixed window sizes $w=3$ and $w=15$ and (c) with the adaptive approach.

computed with the standard matching method using the $M S E^{3}$. Fig. 3 shows the results for the pyramid. The true disparity range for the pyramid is 15 to 28 and for the sphere 15 to 22 pixels. These stereo pairs are tested first with the standard stereo method using fixed window sizes $w=3, w=7$ and $w=15$ and then with the adaptive matching method using the function $C_{\Gamma}$ (Fig. 3). For flat surfaces a large search window obtains good results, whereas on depth edges the disparity values are blurred. The disparity values along depth edges are more accurate using small search windows, but the disparity over the complete image is very noisy. The adaptive approach obtains good results both on depth discontinuities and on flat surfaces. Table 1 illustrates the $\operatorname{MSE}(C(w \in\{3,7,15\})-i d e a l)$ and the $M S E\left(C_{\Gamma}-i d e a l\right)$. The adaptive matching method has the smallest $M S E$.

\begin{tabular}{|c|c|c|c|c|}
\hline MSE & $M S E(C(w=3)-i d e a l)$ & $M S E(C(w=7)-$ ideal $)$ & $M S E(C(w=15)-$ ideal $)$ & $M S E\left(C_{\Gamma}-\right.$ ideal $)$ \\
\hline Pyramid & 280 & 203 & 266 & 50 \\
\hline Sphere & 320 & 280 & 303 & 65 \\
\hline
\end{tabular}

Table 1. Difference between true disparity and the computed disparity.

This approach reduces two types of errors,

- large random errors all over the image caused by a small search window and

- systematic errors along depth discontinuities which occur when using large search windows.

Archaeological fragments are used as real objects $[9,7]$. The experimental setup consists of two 8Bit-CCD cameras with a resolution of $768 \times 568$ pixels and

${ }^{3}$ The notation $M S E(<$ method $>(<w>)-i d e a l)$ is used for the comparison between the ideal and the computed disparity maps. The $M S E$ is only computed for the region which is visible in both stereo images. 


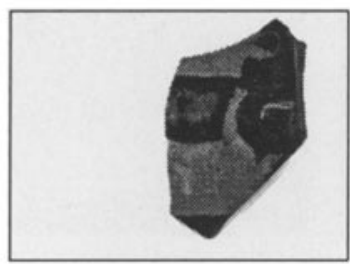

(a) left image

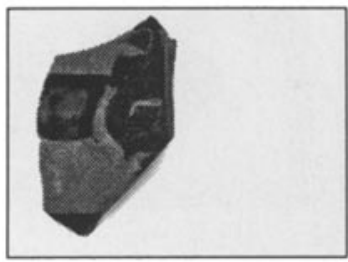

(d) right image

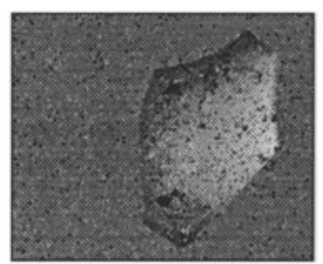

(b) with $C, w=3$

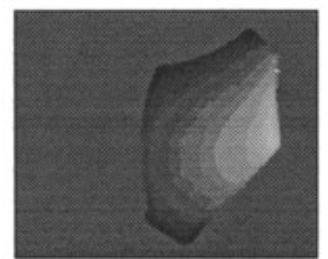

(e) with $C, w=15$

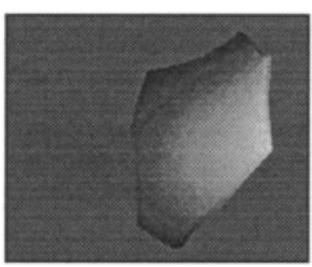

(c) with $C_{\Gamma}$

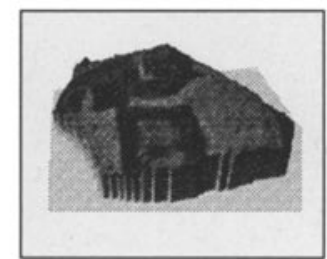

(f) 3D model

Fig. 4. Archaeological fragment 1: (a) and (d) stereo pair, (b)(e) disparity maps computed with the standard method using fixed window sizes $w=3$ and $w=15$ and (c) with the adaptive approach and (f) 3D model of the object.

a 133-Pentium running OS Linux. We tested about twenty different fragments, each of them with different orientation parameters and under varying lighting conditions. The stereo pairs of two different fragments are depicted in Figs. 4 and Figs. 5 (a) and (d).

The curved surface of both fragments is recovered. The objects have a smooth surface without any false matches and the edge of the fragments is also recovered. For comparison the disparity values computed with the standard method using fixed window sizes are shown in Figs. 4 (b),(e) and Figs. 5 (b),(e). A small window size produces several mismatches on the surface of both fragments whereas large search windows smooth the disparity values. Together with both gray-level and disparity information three-dimensional models of the objects with the texture added on the surface can be created(Figs. 4 (f) and 5 (f)).

\section{Summary}

In this paper a new method detects the optimal scale to determine the corresponding region for each location in a given stereo pair. A correlation scale-space defines the scale in a continuous way. For each region in the stereo pair, depending on the gray-level and disparity information, the size of the search window can be changed adaptively in a continuous way by changing the scale parameter $t$ of the correlation scale-space. Furthermore the shape of the search window has changed from rectangular to circular. Experience demonstrates that tilted regions with varying disparity values need a small scale, whereas flat regions with low gray-level variations favor a larger scale which produces a distinct maximum. The global scale-space maximum for a certain region, which maximizes the correlation value is defined as the optimal size of the search window. The adaptive matching strategy combines the benefits of small and large scales 


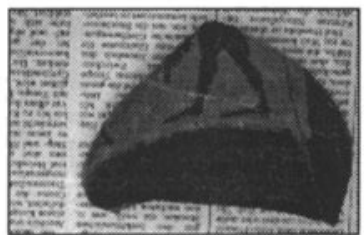

(a) left image

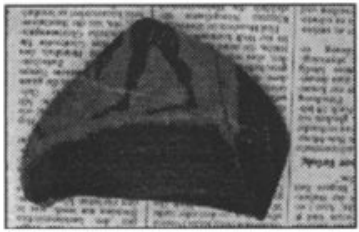

(d) right image

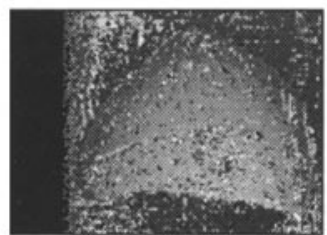

(b) with $C, w=3$

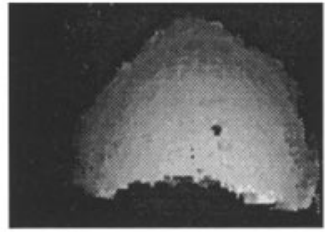

(e) with $C, w=15$

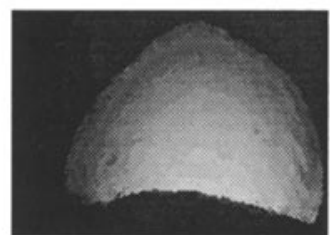

(c) with $C_{\Gamma}$

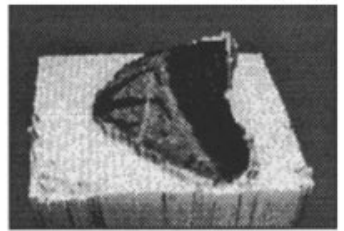

(f) 3D model

Fig. 5. Archaeological fragment 2: (a) and (d) stereo pair (b)(e) disparity maps computed with the standard method using fixed window sizes $w=3$ and $w=15$ and (c) with the adaptive approach and (f) 3D model of the object.

which is demonstrated by experiments with synthetic data and archaeological fragments.

\section{References}

1. S.T. Barnard and M.A. Fischler. Computational stereo. In ACM Computing Surveys, volume 14, pages 553-572, Dec. 1982.

2. M. Jaegersand. Saliency maps and attention selection in scale and spatial coordinates: An information theoretic approach. In Proceedings Fifth Intern. Conf. on Computer Vision, pages 195-202, Cambridge, MA, June 20-23 1995. MIT, IEEE. Catalogue no 95CB35744.

3. T. Kanade and M. Okutomi. A stereo matching algorithm with an adaptive window: Theory and experiment. PAMI, 16(9):920-932, September 1994.

4. M.D. Levine, D.A. O'Handley, and G.M. Yagi. Computer determination of depth maps. Comput. Graphics Image Processing, 2:131-150, September 1973.

5. L. Lindeberg. Scale-Space Theory in Computer Vision. Kluwer Academic Publishers, 1994.

6. C. Menard. Robust Stereo and Adaptive Matching in Correlation Scale-Space. $\mathrm{PhD}$ thesis, TU Wien, Institut für Automation, PRIP, Wien, 1996.

7. C. Menard and R. Sablatnig. Computer based Acquisition of Archaeological Finds: The First Step towards Automatic Classification. In Hans Kamermans and Kelly Fennema, editors, Interfacing the Past. Computer Applications and Quantitative Methods in Archaeology. CAA95, number 28, pages 413-424, Leiden, March 1996. Analecta Praehistorica Leidensia.

8. Azriel Rosenfeld and Avinash C. Kak. Digital Picture Processing Volume 2. Academic Press, Inc, 1982.

9. R. Sablatnig and C. Menard. Stereo and Structured Light as Acquisition Methods in the Field of Archaeology. In New York Springer Verlag Berlin, Heidelberg, editor, Mustererkennung 92 14. DAGM Symposium Dresden, pages 398-404. Fuchs S., 1992.

10. J. Weng. Camera calibration with distortion models and accuracy evaluation. IEEE Trans. Patt. Anal. Machine Intell., 14:965-980, 1992. 